\title{
The Performance Evaluation of a Novel Methodology of Fixational Eye Movements Detection
}

\author{
Jiamao Li and Xiaolin Zhang
}

\begin{abstract}
The vision system plays a very important role for people. Eye movements are an important part of human`s vision system. Fixational eye movements have been studied for many years, but fixational eye movements have not been explained clearly because of the technical problems. To measure these eye movements, we developed a new eye movement measurement device that offers sufficient accuracy. The device can detect rotation of eye movements in three degrees of freedom and is also less-invasive. We conducted some performance evalution experiments in order to validate the feasibility and effectiveness of the system.
\end{abstract}

Index Terms-Fixational eye movements, SURF, sclera image, microsaccades, tremor, drift, high accuracy.

\section{INTRODUCTION}

As we known the vision system plays a very important role for people. Eye movements are an important part of human`s vision system. Scientists have studied eye movements for many years. The human eye movements included cooperative movement, smooth pursuit, saccade, vestibulo-ocular reflex, cervical-ocular reflex and fixational eye movements. [1] Most of the eye movements have been implemented to robots except fixational eye movements. [2] Because people haven't realized the mechanism and theory of this eye movement very clearly.

Actually, our eyes need to move dynamically to project an image onto our retinas during fixation to prevent neural adaptation caused by an unchanging environment. Without this involuntary eye movement, our visual perception would fade completely. In other words, we would be prevented from seeing a stationary object for an extended period. Three different types of eye motion can be detected when we gaze at an object: tremor, drift, and microsaccades (Fig. 1) [3]. The occurrence of several coincident types of movement demonstrates the complexity of the visual system and indicates the need for intensive research. Today, scientists agree that three main types of eye movement occur during visual fixation in humans: tremor, drift, and microsaccades. According to previous experimental results [3] (Fig. 1.), tremor is an aperiodic, wave-like motion of the eyes with a frequency of $\sim 90 \mathrm{~Hz}$, and its amplitude is approximately equal to the diameter of a cone in the fovea[3]. Drift, which is normally superimposed on tremor, is a slow movement with a mean speed of $6 \mathrm{~min} / \mathrm{sec}$ and a maximum speed of $30 \mathrm{~min} / \mathrm{sec}$ The drift frequency is $95 \%-97 \%$ of the fixation time. Its duration is $0.3-0.8 \mathrm{sec}$ [4], and it follows a curved path. The

Manuscript received December 10, 2012; revised February 15, 2013

Jiamao Li is with Department of Information Processing, Tokyo Institute of Technology (e-mail: lijiamao@ipr.pi.titech.ac.jp). third type of eye movement is the microsaccade. These movements have an amplitude of 5-120 min (monocular) or 6-120 min (binocular) and are rapid, with speeds of approximately 6-120 deg/sec (monocular) or 10-120 deg/sec (binocular) [5]. Their motion is jerky.

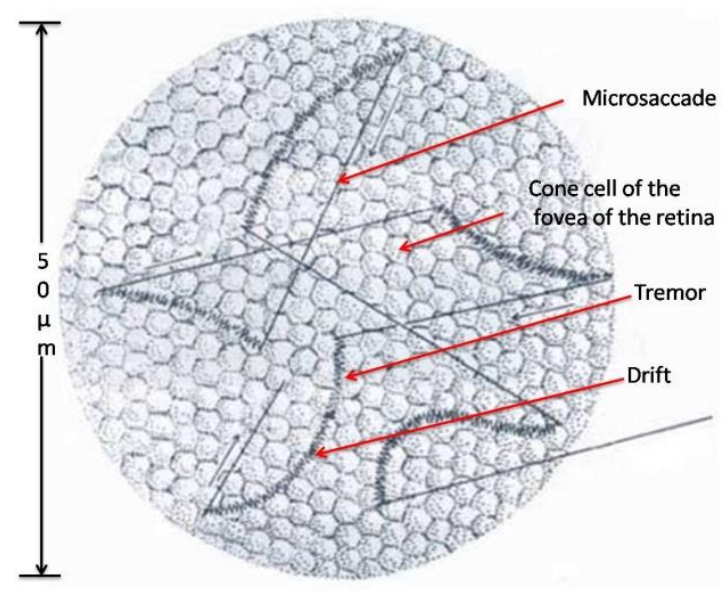

Fig. 1. previous experimental results about fixational eye movements.

Although many methods of detecting these features are available, the classical methodologies are unable to directly capture the smallest eye motions because of their significant technological limitations. The accuracy of devices such as an EOG (Electro-oculogram) [6] or a head-mounted camera [7] is not sufficient to detect the quality of fixational eye movements. Accordingly, Fig. 1 is a schematic diagram rather than an experimental result.To analyse these small movements, a measurement technique needs to be able to detect them in three rotational degrees of freedom simultaneously with an accuracy of $0.0001 \mathrm{deg}$. Moreover, the methodology should be minimally invasive to the experimental subject.

We have developed a new device in our laboratory in response to these demanding requirements. [8]-[10]Here, we use our high-accuracy device to demonstrate several new types of fixational eye movement. These results indicate the need for a review of the conventional thinking about fixational eye movements.

\section{METHOD}

\section{A. System Components}

The eye movement measurement system includes head-fix stage, high-speed cameras, microscope lens, laser line mark, display, optical fiber and vibration removal table. Fig. 2 The head-fix stage was used to fix the head of experimental subject so that the movements of head can be canceled. Two high-speed cameras with microscope lens were used to 
capture the image of blood vessel of white of eye. By using image processing can detect the displacements of the sclera images.The lens we used called telecentric lens. Telecentric lens produces images of the same size regardless of the distance between the lens and image sensor, so we did not need measure the distance between eyeball and camera. We used optical fiber to illuminate because even illumination can be made by it. The display was used to show the experimental target. Laser line marks were used to measure the angle of the camera line.

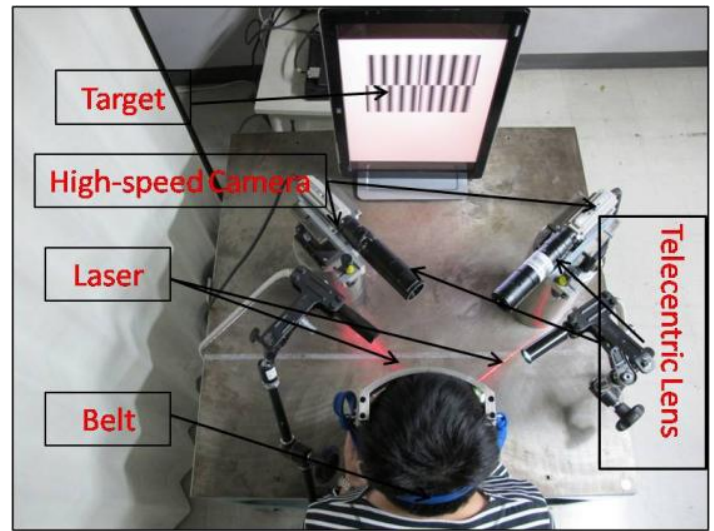

Fig. 2. A photo of the eye movement measurement system (from above).

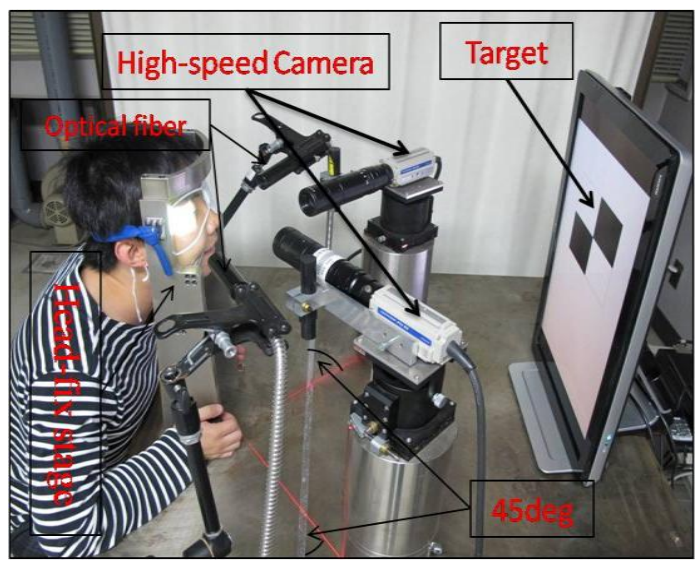

Fig. 2. A photo of the eye movements measurement system(from right).

\section{B. Detection Methods}

Our system was established to measure the three degrees of freedom of eye rotation, as shown in Fig. 3.

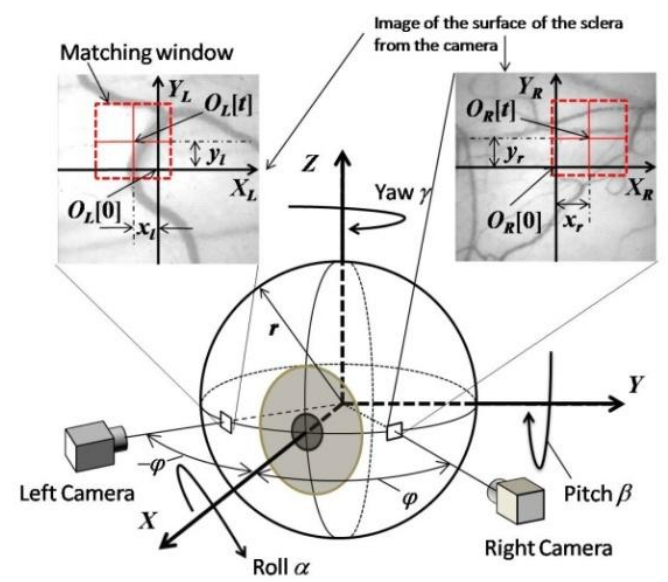

Fig. 3. Definitions of the planes of eye movement.

The displacements $\left(x_{l}, y_{l}\right)$ and $\left(x_{r}, y_{r}\right)$ of the captured eye images shown in Fig. 2 were recorded using an image-processing algorithm, SURF[11], which can perform these measurements in sub-pixels. The displacements $\left(x_{l}, y_{l}\right)$ and $\left(x_{r}, y_{r}\right)$ included both rotational information and translational movements information of the eyeball, i.e.

$$
\begin{aligned}
& x_{l}=x_{l-\gamma}+x_{l-x}+x_{l-y} \\
& y_{l}=y_{l-\alpha}+y_{l-\beta}+y_{l-z} \\
& x_{r}=x_{r-\gamma}+x_{r-x}+x_{r-y} \\
& y_{r}=y_{r-\alpha}+y_{r-\beta}+y_{r-z}
\end{aligned}
$$

where $x_{l-\gamma}$ and $x_{r-\gamma}$ are the displacements caused by rotation $\gamma$, $y_{l-\alpha}$ and $y_{r-\alpha}$ are the displacements caused by rotation $\alpha, y_{l-\beta}$ and $y_{r-\beta}$ are the displacements caused by rotation $\beta, x_{l-x}$ and $x_{r-x}$ are the displacements caused by translational movements in $\mathrm{X}$ axis direction, $x_{l-y}$ and $x_{r-y}$ are the displacements caused by translational movements in Y axis direction, $y_{l-z}$ and $y_{r-z}$ are the displacements caused by translational movements in $\mathrm{Z}$ axis direction.

From the cameras positions, the following equations are obtained.

$$
\begin{aligned}
& x_{l-\gamma}=x_{r-\gamma}, y_{l-\alpha}=-y_{r-\alpha}, y_{l-\beta}=y_{r-\beta}, \\
& x_{l-x}=-x_{r-x}, x_{l-y}=x_{r-y}, y_{l-z}=y_{r-z}
\end{aligned}
$$

So, (1)-(4) the following equation are obtained

$$
\begin{aligned}
& y_{l-\alpha}=-y_{r-\alpha}=\frac{y_{l}-y_{r}}{2} \\
& y_{l-\beta}=y_{r-\beta}=\frac{y_{l}+y_{r}-\left(y_{l-z}+y_{r-z}\right)}{2} \\
& x_{l-\gamma}=x_{l-\gamma}=\frac{x_{l}+x_{r}-\left(x_{l-y}+x_{r-y}\right)}{2}
\end{aligned}
$$

The translational movements of eyeball can be considered caused by the contraction of muscles and the pulsation of blood pressure. From the conformation of human eye, because the eyeball was tightly wrapped in the inside of the eye socket, the translational movements of horizontal ( $Y$ axis direction) and vertical ( $Z$ axis direction) of eyeball are very small. The conformation was only impacted the translational movements on optical axis direction ( $\mathrm{X}$ axis direction), because only the front of eyeball was not wrapped by eye socket. Here we considered that the influence of translational movements of horizontal and vertical can be ignored. Because equation (5)-(7) show that the displacements caused by translational movements on optical axis direction $\left(x_{l-x}\right.$ and $x_{r-x}$ ) are not influence the rotation, therefor the rotation angles of the eyeball can be described by following mathematical equations.

$$
\begin{gathered}
\alpha[t]=\frac{k_{y}}{r \sin \varphi} \cdot \frac{y_{l}[t]-y_{r}[t]}{2} \cdot \frac{180}{\pi} \\
\beta[t]=\frac{k_{y}}{r \cos \varphi} \cdot \frac{y_{l}[t]+y_{r}[t]}{2} \cdot \frac{180}{\pi} \\
\gamma[t]=\frac{k_{x}}{r} \cdot \frac{x_{l}[t]+x_{r}[t]}{2} \cdot \frac{180}{\pi}
\end{gathered}
$$

where $\kappa_{x}$ and $\kappa_{y}$ are the parameters for translating a distant 
point (pixel) on the camera image to a distant imaging area $(\mathrm{mm})$. Because telecentric lenses are used, $\kappa_{x}$ and $\kappa_{y}$ are constant. Parameter $r$ is the radius of the eyeball. Because the value of this parameter is difficult to measure for individual subjects, we have used standard data for the human eye.

$$
\begin{gathered}
\kappa_{x}=\frac{x_{i}}{x_{p}}=\frac{0.842 \mathrm{~mm}}{512}=1.64 \times 10^{-3} \mathrm{~mm} \\
\kappa_{y}=\frac{y_{i}}{y_{p}}=\frac{0.793 \mathrm{~mm}}{480}=1.65 \times 10^{-3} \mathrm{~mm} \\
r=12 \mathrm{~mm}
\end{gathered}
$$

\section{Image Processing}

The system provides accurate results through an image-processing algorithm applied to high-resolution photos of the surface of the sclera. Two high-speed cameras with telecentric lenses were stationed at an identical specified angle from the optical axis of the eye on opposite sides, as illustrated in Fig. 4.

The displacement of the captured eye images was then recorded using an image-processing algorithm, SURF [11], which can perform these measurements in sub-pixels. The approach is described as follows:

1) The first sclera image frame is used as the template frame. SURF extracts a set of feature points from the template.

2) Extract the same feature points in the reference frames, and match the feature points in the template and reference frames.

3) Delete the incorrectly matched feature points. The feature points in the template frames should have the same relative positions as in the template frame.

4) Calculate the displacement between the reference and template frames using the positions of the feature points in the two frames.

\section{EXPERIMENTS}

We conducted some performance evalution experiments in order to validate the feasibility and effectiveness of the system.

\section{A. Error of Image Processing}

In order to detect the accuracy of image processing, we conducted the verification experiment. The detection system which can measure the fixational eye movements should have the accuracy by sub-pixel. Here we calculated the error of the program we made.

First, we made a simulation video which we known the true value of the displacement in the images. In a $512 \times$ 480pixel sclera image, we moved each 0.5 pixel to cut a vector of $452 \times 420$ pixel. Every vector was one frame of the simulation video.

The the true value and the detected value of the simulation video was shown in Fig. 4 a and Fig. 4 b.

Fig. 5 shown the errors of the tracking processing in horizontal and vertical. All the errors were small than 0.1 pixel. The result shown that the method we used in detecting the displacement of sclera images has enough accuracy to the fixational eye movements measurement.
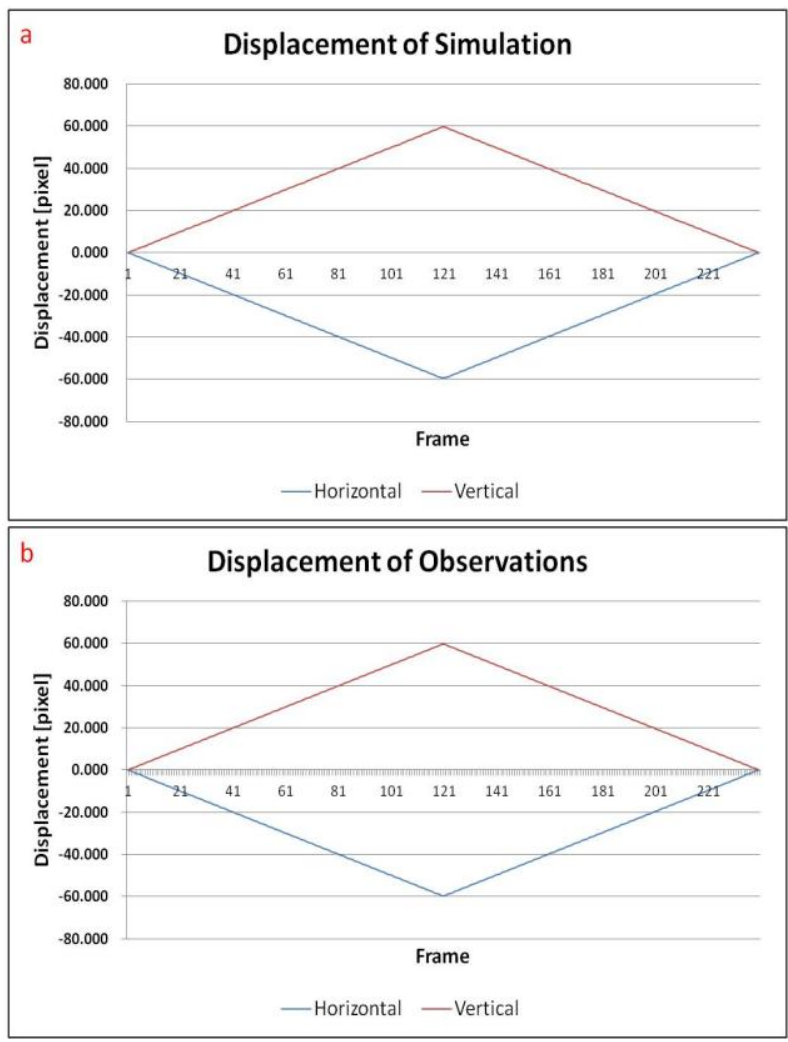

Fig. 4. The true value and the detected value of the simulation video.
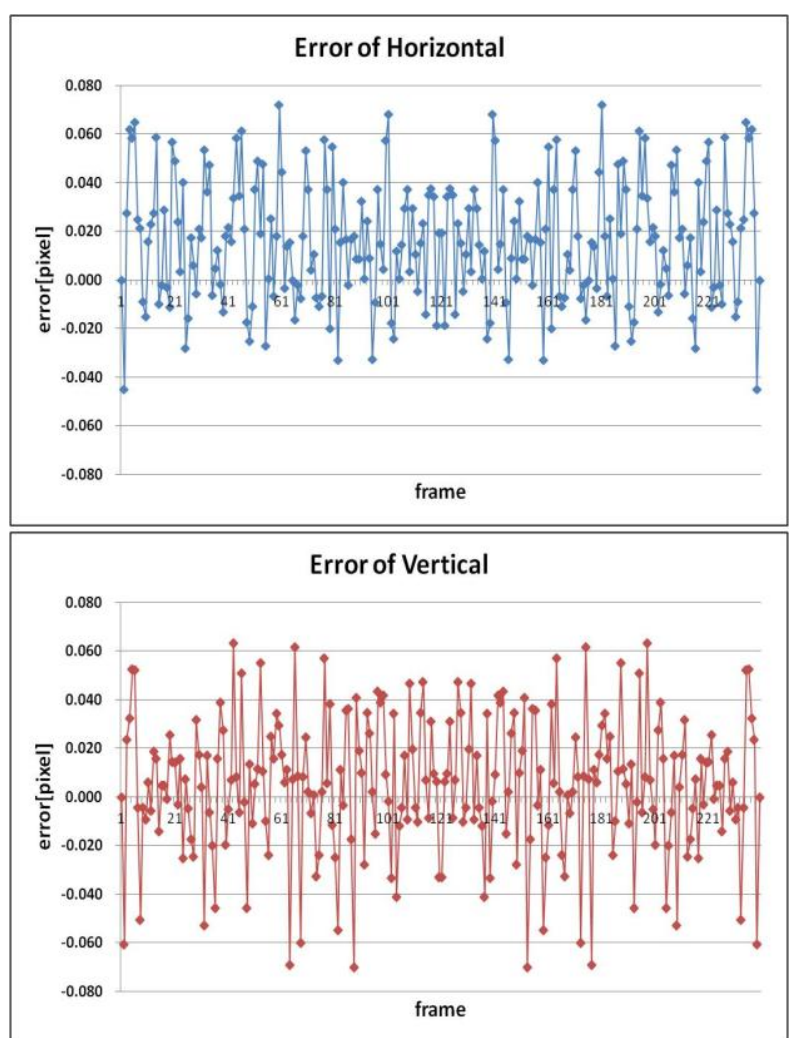

Fig. 5. Error of image processing.

\section{B. Infinitesimal Motion of the Head.}

We conducted the verification experiment in order to detect the effects of the movement of head. We detected the movement of head when the head of experimental subject was fixed same as the fixational eye movements measurement experiments. 


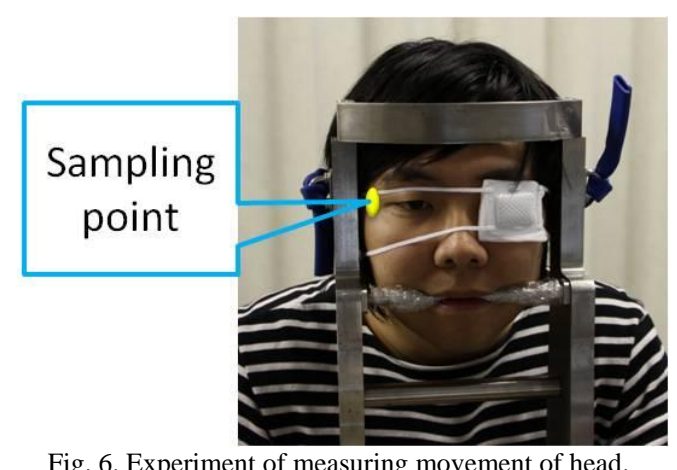

Fig. 6. Experiment of measuring movement of head.

As shown in Fig. 6, We used high-speed camera to capure the canthal skin when the subject's head was fixed to the stage. The magnification of the lens was 4.5 times, the frame rate was set as 250fps. Image processing was used to calculate the displacement of capture area. The result was shown in Fig. 7 a). Although it is difficult to completely remove the effect of the head movement, comparing with the displacement of fixational eye movements Fig. 7 b), the effect was very small.

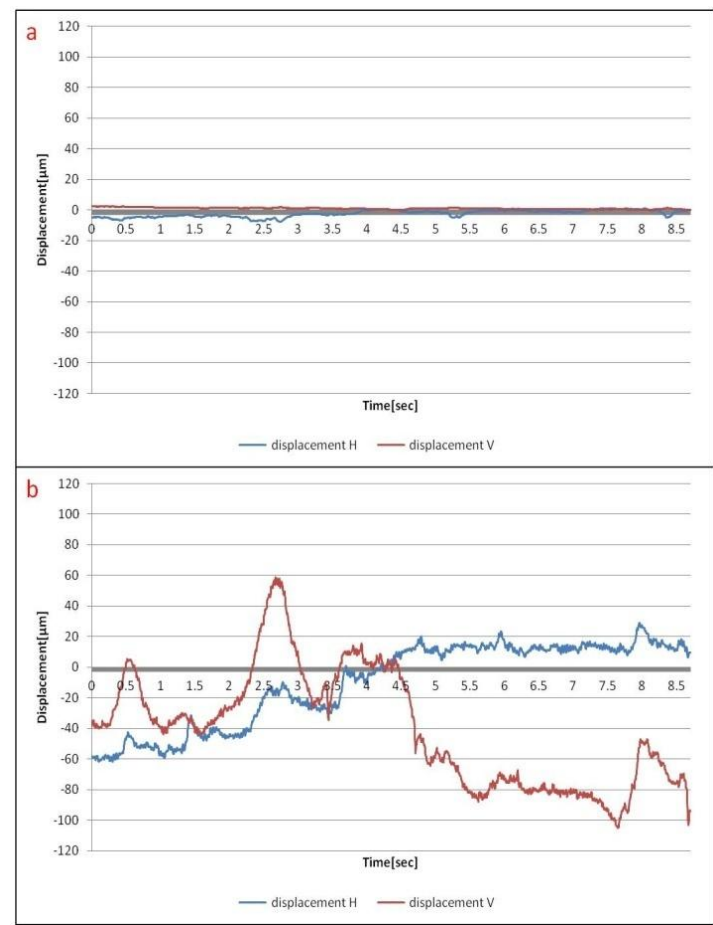

Fig. 7. a) Displacement of head movement; b) Displacement of fixational eye movements.

\section{Performance Compared with Existing Methods}

TABLE I: PERFORMANCE OF SEVERAL Eye-RECORDING METHOD [12], [13]

\begin{tabular}{cccc}
\hline & $\begin{array}{c}\text { Eyelink 1000 } \\
\text { (SR } \\
\text { Research) }\end{array}$ & $\begin{array}{c}\text { EMR-9 } \\
\text { (nac) }\end{array}$ & $\begin{array}{c}\text { Sclera vessel } \\
\text { tracking } \\
\text { method } \\
\text { (Zhang Lab) }\end{array}$ \\
\hline $\begin{array}{c}\text { Spatial } \\
\text { resolution }\end{array}$ & $0.01^{\circ}$ & $\begin{array}{c}\text { (H) } 0.1^{\circ} \\
\text { (V) } 0.4^{\circ}\end{array}$ & $0.0008^{\circ}$ \\
\hline $\begin{array}{c}\text { Temporal } \\
\text { resolution }\end{array}$ & $1000 \mathrm{~Hz}$ & $240 \mathrm{~Hz}$ & $1000 \mathrm{~Hz}$ \\
$\begin{array}{c}\text { Detection } \\
\text { method }\end{array}$ & $\begin{array}{c}\text { Corneal } \\
\text { reflection } \\
\text { method }\end{array}$ & $\begin{array}{c}\text { Corneal } \\
\text { reflection } \\
\text { method }\end{array}$ & $\begin{array}{c}\text { Sclera vessel } \\
\text { tracking } \\
\text { method }\end{array}$ \\
$\begin{array}{c}\text { Rotation } \\
\text { around } \\
\text { visual line }\end{array}$ & OK & Impossible & OK \\
\hline
\end{tabular}

\section{Application of the System}

We found an application of this fixational eye movements measurement system, that is the relationship between fixational eye movements and human's mental state. The muscles which controls the eye movements have been classified as voluntary muscles. The movements of heart was controlled by the voluntary muscles. The physical condition of human can be checked by using ECG. Thus, detecting fixational eye movements may check the state of brain, because eyeball is the only organ connected to brain, which extends to outside of the body. We compared the fixational eye movements in normal state and in thinking state, in order to find the pattern of fixational eye movements which was associated with psychological state. We found that when people were in thinking, the number of microsaccades in the unit of time was reduced. Fig. 8 is an example of one person`s fixational eye movements in normal state and in thinking state.

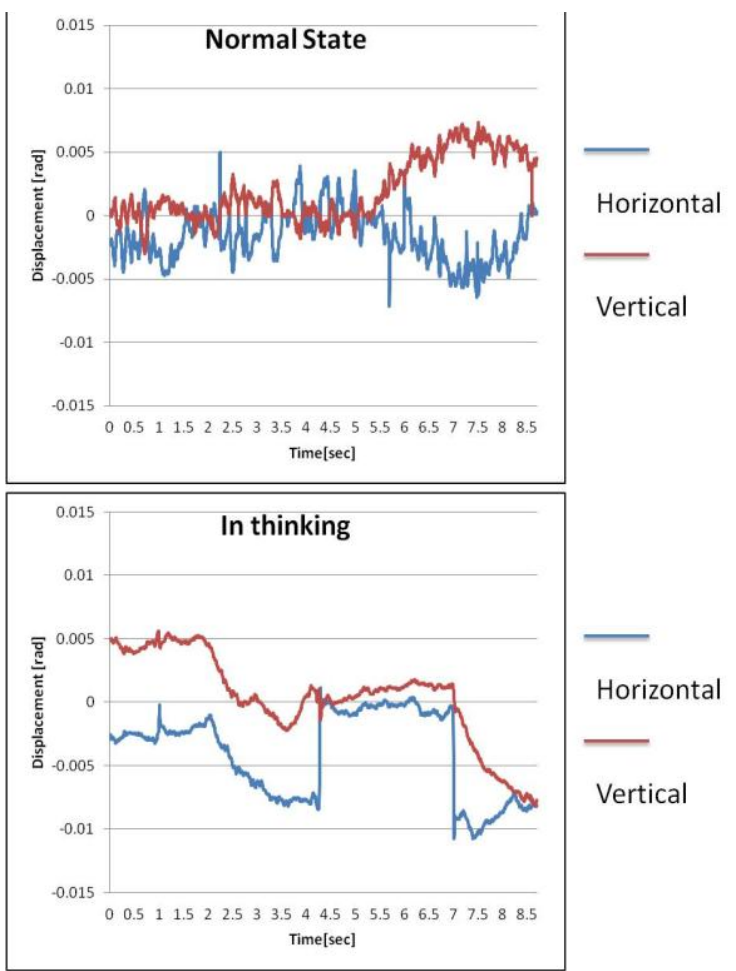

Fig. 8. The fixational eye movements in normal state and in thinking state.

\section{CONCLUSION}

To measure fixational eye movements, we developed a new eye movement detection system based on sclera vessels tracking method that offers sufficient accuracy.

This device can detect rotation in three degrees of freedom simultaneously and is also non-invasive.

The system has spatial resolution of $0.0008 \mathrm{deg}$ and temporal resolution of $1000 \mathrm{~Hz}$.

This system not only can measure fixational eye movements but also can be used in ophthalmic diagnostic, blood examination [14], psychology and so on.

\section{ACKNOWLEDGMENT}

This work was partly supported by Panasonic. We also thank Ms. Kiyomi SAKAMOTO, Mr. Shoichi AOYAMA, and Mr. Shigeo ASAHARA for their support and discussion. 


\section{REFERENCES}

[1] X. L. Zhang. Homepage of Zhang Lab. [Online]. Available: http//www.zhang.pi.titech.ac.jp

[2] X. L. Zhang, "An object tracking system based on human neural pathways of binocular motor system," in Proc. 9th Int. Conf. Control, Automation, Robotics and Vision, Singapore, December 2006, pp. 285-292.

[3] S. M. Conde, S. L. Macknik, and D. H. Hubel, "The Role of Fixational Eye Movements in Visual Perception," Nature Reviews. Neuroscience, vol. 5, no. 3, pp. 229-240, Nature Publishing Group, London, 2004.

[4] A. L. Yarbus, Eye Movements and Vision, pp. 107-113, 1967.

[5] R. Engbert and R. Kliegl, In the Mind's Eyes: Cognitive and Applied Aspects of Eye Movements, pp. 103-117, 2003.

[6] B. Tursky, "Recording of Human Eye Movements," Bioelectric Recording Techniques Part C, New York: Academic Press, 1974.

[7] S. Yusuke, F. Hironobu, and H. Yutaka, "Measurement of 3D eye movements using image processing," vol.6, no. 3, pp. 236-243, 2006.

[8] X. L. Zhang and J. M. Li, "A novel methodology for high accuracy fixational eye movements detection," in Proc. 4th International Conference on Bioinformatics and Biomedical Technology, Singapore, Feb, 2012, pp. 133-140.

[9] J. M. Li and X. L. Zhang, "Novel human fixational eye movements detection using sclera images of the eyeball," Japanese Society of Clinical Physiology, vol. 42, no. 3, 2012.

[10] J. M. Li and X. L. Zhang, "Using high-speed photography and image processing for fixational eye movements measurement," in Proc. 2012 IEEE International Conference on Imaging Systems and Techniques, 16-17, July, 2012, Manchester, UK, pp. 28-33.

[11] H. Bay, T. Tuytelaars, and L. V. Gool, "SURF: Speeded Up Robust Features," in Proc. 9th European Conference on Computer Vision, LNCS, Springer, vol. 3951, part 1, 2006, pp. 404-417.

[12] Manual of EyeLink 1000. [Online]. Available: http://www.sr-research.com
[13] Manual of EMR-9. [Online]. Available: http://www.nacinc.jp/

[14] J. M. Li and X. L. Zhang, "A Novel Method for Blood Flow Measurement based on Sclera Images," in Proc. 4th International Conference on Bioinformatics and Biomedical Technology, Singapore, Feb. 2012, pp. 74-81.

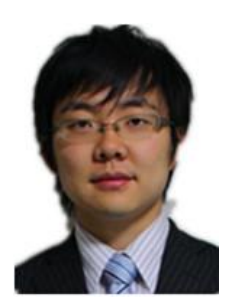

Jiamao Li was born in Wuxi, China, in 1983. He received the M.S. degree in Department of Information Processing from Tokyo Institute of Technology, Japan, in 2010. He is currently a student of doctor course of Department of Information Processing, Tokyo Institute of Technology. His research interests include image processing and biomedical engineering.

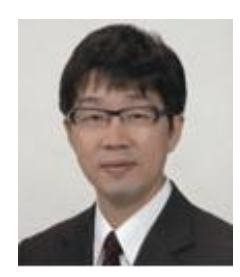

Xiaolin Zhang was born in Jilin, China, in 1963. He received B.E. in department of power systems a Northeast China Institute of Power Engineering in 1985. He received M.S.E. in mechanical engineering at Yokohama National University in 1989. He was a research associate in engineering at Yokohama National University during 1989-1992. He received the Eng.D at Yokohama National University in 1995. $\mathrm{He}$ was an assistant professor in biomedical engineering at Tokyo Medical and Dental University during 1995-2003. He was an associate professor in precision and intelligence laboratory at Tokyo institute of technology during 2003-2012.

$\mathrm{He}$ is currently a professor in precision and intelligence laboratory, Tokyo Institute of Technology. His research interests include bio-robotics, intelligence system and biomedical engineering. 